\title{
Shot-Noise Seeded Microbunching Instability: Second-Order Correction to the Gain Function
}

\author{
Marco Venturini \\ Lawrence Berkeley National Laboratory, University of California, Berkeley, California, 94720
}

\begin{abstract}
We determine the second-order correction to the gain function of the microbunching instability in single-pass systems of interest for the next generation of light sources. The calculation applies to the case where the instability is seeded by shot noise. We examine an analytically treatable model of beam dynamics where collective forces are active only in non-dispersive sections of the linac. We find that the second order term can augment the linear gain significantly while affecting the spectrum of the overall gain only marginally. The weight of the second-order correction relative to the linear gain is found to scale quadratically with respect to $R_{56}$. The qualitative behavior predicted by the model is consistent with exact numerical solutions of the Vlasov equations for realistic lattices.
\end{abstract}

Key words:

PACS:

\section{Introduction}

The microbunching instability can be an important factor limiting beam quality and hence performance of FEL-based 4th generation light sources [1-8]. The drive linac for a single-pass FEL acts as a noise amplifier for density modulations present in the beam at injection. The noise amplification results from collective effects (mostly longitudinal space-charge) and the non-zero dispersion generated by the magnetic chicanes used for bunch compression. The gain function for this instability is fairly broadband and delimited at small wavelengths by phase-space mixing due to finite beam energy spread and transverse emittance.

An estimate of the gain function through a chicane for a 1D model of beam and simplified dynamics was first reported in [2]. Further refinements have allowed for account of a finite transverse emittance and a more comprehensive inclusion of collective effects $[3,4]$. The analysis in the latter references is limited to the linear approximation, where 'linear' means first order with respect to the strength the interparticle forces. The analysis in [2] includes nonlinearities but takes into consideration only a single initial sinusoidal perturbation to the beam density. For typical 4th generation light-source parameters nonlinear corrections to the gain function for single sinusoidal modes are usually irrelevant. However, nonlinear corrections can be important if the spectrum of the perturbation is broad band. We came across this realization in our studies of the microbunching instability using a recently developed Vlasov solver $[9,8,10]$. We have been interested in characterizing the effect of shot noise, the fluctuations in the beam density due to the granularity of the elementary charge. Under certain conditions we found that amplification of shot-noise could be a few times larger than predicted by linear theory.

Because of its nonlinear nature an accurate modelling of this phenomenon requires recourse to numerical methods. However, we can gain useful in- 
sight by considering a simplified but analytically treatable model of beam dynamics. The model we discuss in this paper is similar to that considered in [2] but is extended to include the case of instabilities seeded by density perturbations with uniform power spectrum.

The possibility that the gain function for the microbunching instability can be enhanced by nonlinearities is easier illustrated by considering the presence of just two modes in the noise spectrum, say with wavenumbers $k_{1}$ and $k_{2}$. Assuming that the beam has a gaussian energy spread with rms relative energy $\sigma_{\delta}$ linear theory indicates that the amplitude of a mode with wavenumber $k$ is exponentially suppressed at high frequency by a factor $\exp \left(-C^{2} k^{2} R_{56}^{2} \sigma_{\delta}^{2} / 2\right)$ where $R_{56}$ is the entry of the transfer matrix relating longitudinal slippage to energy deviation and $C$ is the compression factor through the dispersion region. Suppose that $C k R_{56} \sigma_{\delta} \gg 1$ for both $k=k_{1}$ and $k=k_{2}$. Linear theory would predict an effectively complete damping of both modes. However, second-order coupling generates harmonics with wavenumbers $k_{1}+k_{2}$ and $k_{1}-k_{2}$. If $k_{1}$ and $k_{2}$ are sufficiently close that $C\left|k_{1}-k_{2}\right| R_{56} \sigma_{\delta}$ is of the order of unity the mode with frequency $k_{1}-k_{2}$ will escape exponential attenuation and can appear in the bunch spectrum at the end of the dispersive region. A more detailed discussion of this simple two-harmonic model is sketched in Sec. 3. An estimate of the gain function through second order for perturbations with uniform power spectrum is worked out in Sec. 4. A discussion of a numerical example follows in Sec. 5.

\section{The model}

We consider a model of linac consisting of a straight section of length $L_{s}$ followed by a dispersive region with the bunch compressor. We denote with $s_{0}$ the arclength coordinate at the start of the straight section and $s_{1}=s_{0}+L_{s}$ the coordinate at the start of the dispersive region. In the numerical examples we discuss in Sec. 5 the dispersive region will consist of two chicanes but in the analytical calculation one need not be specific regarding the layout of the magnetic compressor as our results will depend only on $R_{56}$ at the observation point. A simplifying assumption is to include only the effect of collective forces in the straight channel while neglecting those originating within the dispersive region. This is not unreasonable as in practical cases the contribution from the latter tends indeed to be smaller but this assumption will lead to some underestimate of microbunching. We also assume a 2D (longitudinal) model of beam phase space neglecting the dynamical effects due to a finite transverse emittance. However, this does not add further simplification as a finite beam transverse emittance plays a role in the dynamics of microbunching only if collective effects in the dispersive region are accounted for. The transverse beam size enters into the model only through determination of the longitudinal electric field due to space-charge.

At the start of the straight section $s=s_{0}$ the beam has a gaussian density in energy

$f\left(z, \delta ; s_{0}\right)=N_{b} \rho\left(z ; s_{0}\right) \frac{e^{-\left[\delta-h_{0} z\right]^{2} / 2 \sigma_{\delta}^{2}}}{\sqrt{2 \pi} \sigma_{\delta}}$,

with rms relative energy spread $\sigma_{\delta}$. In the above equation $N_{b}$ is the number of particles in the bunch, $h_{0}$ is the initial energy chirp. The beam density is (on average) a flat top with length $L_{b}$, i.e. $\rho(z)$ consists of a uniform part $\rho_{0}=1 / L_{b}$ plus a random zero-average perturbation. The normalization is $\int_{-L_{b} / 2}^{L_{b} / 2} \rho\left(z ; s_{0}\right) d z=1$. We will denote the linear particle density as $n_{b}=N_{b} / L_{b}$. We assume periodic boundary conditions and write the longitudinal density as a Fourier series $\rho\left(z ; s_{0}\right)=\sum_{n=-\infty}^{\infty} \tilde{\rho}_{n}\left(s_{0}\right) e^{i k_{n} z}$ with $\tilde{\rho}_{n}\left(s_{0}\right)=$ $L_{b}^{-1} \int_{-L_{b} / 2}^{L_{b} / 2} e^{-i k_{n} z} \rho\left(z ; s_{0}\right) d z$ and wavenumbers $k_{n}=$ $2 \pi n / L_{b}$. Assuming ultrarelativistic dynamics (relativistic factor $\gamma \gg 1$ ) the longitudinal density $\rho(z)$ can be regarded as 'frozen' as the beam travels through the straight section between $s_{0}$ and $s_{1}$. By the time the beam reaches the entry of the dispersive region at $s_{1}$ the random perturbations in the charge density have generated the following $z$-dependent relative energy modulation

$g(z)=\sum_{n=-\infty}^{\infty} \tilde{g}_{n} e^{i k_{n} z}$

with

$\tilde{g}_{n}=-\frac{e^{2} N_{b} L_{s}}{m c \gamma} Z\left(k_{n}\right) \tilde{\rho}_{n}\left(s_{0}\right)$

where $\gamma$ is the relativistic factor of the beam at $s=$ $s_{1}$ and $Z\left(k_{n}\right)$ is an impedance per unit length describing the collective forces.

For the impedance we assume the model of longitudinal space charge $[11,7,12]$ : 


$$
Z(k)=\left.\frac{i Z_{0}}{\pi \gamma_{*} r_{b}} \frac{1-\xi K_{1}(\xi)}{\xi}\right|_{\xi=k r_{b} / \gamma_{*}},
$$

where $Z_{0}=\left(\varepsilon_{0} c\right)^{-1} \simeq 120 \pi \Omega$ is the vacuum impedance and $K_{1}$ the modified Bessel function. This model applies to a beam with uniform transverse density and circular cross section of radius $r_{b}$. A more accurate modelling would require integrating (4) with respect to energy to account for possible acceleration through the straight section. In the following we will content ourselves with using the space charge impedance in the form (4) with relativistic factor $\gamma$ chosen to be an effective value $\gamma=\gamma_{*}$, between the values of $\gamma$ before and after acceleration.

\section{One and two-harmonic perturbation of initial beam density}

Here we are interested in the Fourier spectrum of the beam longitudinal density evolving from an initial beam charge density seeded with one or two sinusoidal perturbations.

As the beam travels through the straight section from $s_{0}$ to $s_{1}$ the density function in the longitudinal phase space evolves to $f\left(z, \delta ; s_{1}\right)=f\left(z, \delta-g(z) ; s_{0}\right)$, where $f\left(z, \delta ; s_{0}\right)$ is given by Eq. (1). ${ }^{1}$ Next, as the beam travels into the dispersion region $s>s_{1}$ the density evolves to $f(z, \delta ; s)=f\left(z-R_{56} \delta, \delta ; s_{1}\right)$. We can then compute $\rho(z ; s)=\int_{-\infty}^{\infty} d \delta f(z, \delta ; s)$ and find for $s>s_{1}$

$$
\begin{aligned}
\rho(z ; s) & \simeq C \sum_{n \neq 0} \tilde{\rho}_{n} e^{i C k_{n} z} e^{-\left(\bar{R}_{56} C k_{n}\right)^{2} / 2} \\
& +\frac{C \rho_{0}}{\sqrt{2 \pi}} \int_{-\infty}^{\infty} d t e^{-\left[t-\bar{g}\left(z C-\bar{R}_{56} t C\right)\right]^{2} / 2},
\end{aligned}
$$

where $C=1 /\left(1-h_{1} R_{56}\right)$ is the compression factor and to keep notation compact we have introduced the scaled quantities $\bar{R}_{56}=R_{56} \sigma_{\delta}$, and $\bar{g}(z)=$ $g(z) / \sigma_{\delta}$. In cases of interest the first term on the RHS is generally small and will be neglected in our analysis.

Suppose the initial perturbation to the beam density consists of a single sinusoidal perturbation with wavenumber $k=k_{m}$ generating an energy modulation $\bar{g}(z)=A \sin (k z)$ by the time the beam reaches the beginning of the dispersion region. The integral

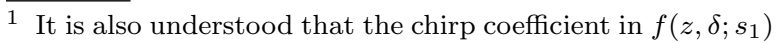
is $h_{1}$, generally different from $h_{0}$.
}

in the second term in the RHS of (5) can be represented as a Fourier series yielding [2]

$\rho(z ; s)=C \rho_{0}\left[1+2 \sum_{n=1}^{\infty} J_{n}(n \alpha) e^{-n^{2} \beta^{2} / 2} \cos (n C k z)\right]$,

where $J_{n}$ is the Bessel function, $\beta=C k \bar{R}_{56}$ and $\alpha=A \beta$. The above formula shows generation of an infinite spectrum of harmonics. However, for large $k$, or $\beta \gg 1$, higher order harmonics are effectively suppressed and only the $n=1$ harmonic is significant. In cases of interest it is usually $\alpha \ll 1$ and therefore $\rho(z ; s) \simeq C \rho_{0}\left[1+A \beta e^{-\beta^{2} / 2} \cos (C k z)\right]$.

Now, suppose that the initial perturbation consists of two harmonics with wavenumbers $k$ and $k^{\prime}$ and same amplitude $\bar{g}(z)=A \sin (k z)+A \sin \left(k^{\prime} z\right)$. Through second order in $A$ :

$$
\begin{aligned}
\int_{-\infty}^{\infty} d t e^{-(t-\bar{g})^{2} / 2} & =\sqrt{2 \pi}-\int_{-\infty}^{\infty} t e^{-t^{2} / 2} \bar{g} d t \\
& +\frac{1}{2} \int_{-\infty}^{\infty}\left(t^{2}-1\right) e^{-t^{2} / 2} \bar{g}^{2} d t+\mathcal{O}\left(\bar{g}^{3}\right) .
\end{aligned}
$$

The resulting first-order term for $\rho(z ; s)$ reads $C \rho_{0} A\left[\exp \left(-\beta^{2} / 2\right) \cos (C k z)+\exp \left(-\beta^{\prime 2} / 2\right) \cos \left(C k z^{\prime}\right)\right]$, where $\beta=C k \bar{R}_{56}$ and $\beta^{\prime}=C k^{\prime} \bar{R}_{56}$, and follows from linear superposition of the first-order singleharmonic spectra. As for the second-order term we have

$$
\begin{aligned}
& \frac{A^{2}}{4}\left[(2 \beta)^{2} e^{-(2 \beta)^{2} / 2} \cos (2 C k z)\right. \\
+ & \left(2 \beta^{\prime}\right)^{2} e^{\left.-\left(2 \beta^{\prime}\right)^{2}\right) / 2} \cos \left(2 C k z^{\prime}\right) \\
+ & \left(\beta+\beta^{\prime}\right)^{2} e^{-\left(\beta+\beta^{\prime}\right)^{2} / 2} \cos \left[C\left(k+k^{\prime}\right) z\right] \\
+ & \left(\beta-\beta^{\prime}\right)^{2} e^{-\left(\beta-\beta^{\prime}\right)^{2} / 2} \cos \left[C\left(k-k^{\prime}\right) z\right] .
\end{aligned}
$$

Observe that if $\beta \gg 1$ and $\beta^{\prime} \gg 1$ the first-order terms as well the first three terms in the expression (7) are exponentially small while the last term in (7) can be significant if $\beta-\beta^{\prime}$ is of the order of unity. We conclude that that perturbation harmonics that would individually be first-order suppressed can still contribute to the low-frequency region of the spectrum through nonlinear coupling.

\section{Amplification of Shot Noise}

We now consider the case of a beam density perturbation with broad spectrum. More specifically, 
we are interested in the evolution of the fluctuations in the initial beam density due to the granularity of the elementary charge (shot noise). The noise (see Appendix A) is delta-correlated, $\left\langle\left[\rho\left(z, s_{0}\right)-\right.\right.$ $\left.\left.\rho_{0}\right]\left[\rho\left(z^{\prime}, s_{0}\right)-\rho_{0}\right]\right\rangle=\left(N_{b} L_{b}\right)^{-1} \delta\left(z-z^{\prime}\right)$ yielding the uniform power spectrum, $\left\langle\tilde{\rho}_{n} \tilde{\rho}_{m}\right\rangle^{1 / 2}=\delta_{n m} \tilde{\rho}$, where $\tilde{\rho} \equiv\left(L_{b} \sqrt{N_{b}}\right)^{-1}=\rho_{0} / \sqrt{N_{b}}$. In the above expressions $\langle\cdot\rangle$ represents averaging over realizations of the the noise.

Having determined the Fourier coefficients of the beam density $\rho(z ; s)$ at $s$

$\tilde{\rho}_{n}(s)=\frac{C}{L_{b}} \int_{-L_{b} / 2 C}^{L_{b} / 2 C} e^{-i C k_{n} z} \rho(z ; s) d z$

we define the amplification (or gain) function for the microbunching as the ratio

$\mathcal{G}\left(k_{n}\right)=\frac{\left\langle\left|\tilde{\rho}_{n}(s)\right|^{2}\right\rangle^{1 / 2}}{C \rho_{0}} / \frac{\tilde{\rho}}{\rho_{0}}$.

In the definition (8) notice the presence of the compression factor $C$, inserted in order to express our results in terms of the wavenumbers before compression. It also accounts for compression of the bunch length to $L_{b} / C$.

In preparation for the calculation of the gain function through second order we write $\rho(z ; s) \simeq$ $\rho^{(0)}(z ; s)+\rho^{(1)}(z ; s)+\rho^{(2)}(z ; s)$ as the sum of a zero-order term (which will be irrelevant for us) and the first-order $\rho^{(1)}(z ; s)$ and second-order $\rho^{(2)}(z ; s)$ terms

$$
\begin{aligned}
& \rho^{(1)}=-\frac{C \rho_{0}}{\sqrt{2 \pi}} \int_{-\infty}^{\infty} t e^{-t^{2} / 2} \bar{g}\left(z C-\bar{R}_{56} t C\right) d t, \\
& \rho^{(2)}=\frac{C \rho_{0}}{2 \sqrt{2 \pi}} \int_{-\infty}^{\infty}\left(t^{2}-1\right) e^{-t^{2} / 2} \bar{g}^{2}\left(z C-\bar{R}_{56} t C\right) d t .
\end{aligned}
$$

\subsection{Linear Gain}

From (8), (10) and (2) we find

$$
\tilde{\rho}_{n}^{(1)}(s)=i C \rho_{0}\left(R_{56} C k_{n}\right) e^{-\left(C k_{n} \bar{R}_{56}\right)^{2} / 2} \tilde{g}_{n},
$$

which leads to the first-order gain function

$$
\mathcal{G}^{(1)}\left(k_{n}\right)=\frac{\left\langle\left|\tilde{\rho}_{n}^{(1)}(s)\right|^{2}\right\rangle^{1 / 2}}{C \tilde{\rho}}
$$

$$
=\frac{e^{2} n_{b} L_{s}}{m c \gamma} Z\left(k_{n}\right) R_{56} C k_{n} e^{-\left(C k_{n} \bar{R}_{56}\right)^{2} / 2},
$$

having made use of

$\left\langle\left|\tilde{g}_{n}\right|^{2}\right\rangle^{1 / 2}=\frac{e^{2} N_{b} L_{s}}{m c \gamma}\left|Z\left(k_{n}\right)\right| \tilde{\rho}$.

Specialized to the impedance (4) the gain reads $\mathcal{G}^{(1)}=\frac{4 I L_{s}}{I_{A} \gamma r_{b}} \frac{1-\xi_{n} K_{1}\left(\xi_{n}\right)}{\gamma_{*} \xi_{n}} R_{56} C k_{n} e^{-\left(\bar{R}_{56} C k_{n}\right)^{2} / 2}$,

where $\xi_{n}=k_{n} r_{b} / \gamma_{*}, I=e c n_{b}$ is the peak current before compression, and $I_{A}=e c / r_{e} \simeq 17 \mathrm{kA}$ the Alfvén current, where $r_{e}$ is the electron classical radius.

\subsection{2nd-Order Correction to Gain}

Evaluation of the FT of the second-order term of the density function (11) yields

$\tilde{\rho}_{n}^{(2)}(s)=-\frac{C \rho_{0}}{2}\left(R_{56} C k_{n}\right)^{2} e^{-\left(C k_{n} \bar{R}_{56}\right)^{2} / 2} \sum_{m=-\infty}^{\infty} \tilde{g}_{m} \tilde{g}_{n-m}$.

To determine the gain function we need $\left\langle\tilde{\rho}_{n}(s) \tilde{\rho}_{m}^{*}(s)\right\rangle$. Through second order $(n, m \neq 0)$

$$
\begin{aligned}
\left\langle\tilde{\rho}_{n}(s) \tilde{\rho}_{m}^{*}(s)\right\rangle & =\left\langle\tilde{\rho}_{n}^{(1)}(s) \tilde{\rho}_{m}^{(1) *}(s)\right\rangle+\left\langle\tilde{\rho}_{n}^{(1)}(s) \tilde{\rho}_{m}^{(2) *}(s)\right\rangle \\
& +\left\langle\tilde{\rho}_{n}^{(2)}(s) \tilde{\rho}_{m}^{(1) *}(s)\right\rangle+\left\langle\tilde{\rho}_{n}^{(2)}(s) \tilde{\rho}_{m}^{(2) *}(s)\right\rangle .
\end{aligned}
$$

The first term on the RHS of the above equation vanishes for $n \neq m$ and for $n=m$ was calculated in Eq. (13). The second and third terms vanish. As for the fourth term:

$$
\begin{aligned}
\left\langle\tilde{\rho}_{n}^{(2)}(s) \tilde{\rho}_{m}^{(2) *}(s)\right\rangle & \propto\left\langle\sum_{p} \tilde{g}_{p} \tilde{g}_{n-p} \sum_{\ell} \tilde{g}_{\ell}^{*} \tilde{g}_{m-\ell}^{*}\right\rangle \\
& =\delta_{n m} \sum_{p}\left\langle\left|\tilde{g}_{p}\right|^{2}\right\rangle\left\langle\left|\tilde{g}_{n-p}\right|^{2}\right\rangle .
\end{aligned}
$$

Having made use of (14) we can then write

$$
\begin{aligned}
\left\langle\left|\tilde{\rho}_{n}^{(2)}(s)\right|^{2}\right\rangle= & {\left[\mathcal{G}^{(1)}\left(k_{n}\right)\right]^{2} C^{2}\left[\frac{e^{2} N_{b} L_{s}}{2 m c \gamma}\right]^{2}\left(R_{56} C k_{n}\right)^{2} \tilde{\rho}^{4} } \\
& \times \frac{\sum_{p}\left|Z\left(k_{p}\right)\right|^{2}\left|Z\left(k_{n}-k_{p}\right)\right|^{2}}{\left|Z\left(k_{n}\right)\right|^{2}}
\end{aligned}
$$

We replace the mode summation over the impedance with an integral 
$\sum_{p}\left|Z\left(k_{p}\right)\right|^{2}\left|Z\left(k_{p}-k_{n}\right)\right|^{2} \simeq \frac{L_{b}}{2 \pi}\left(\frac{Z_{0}}{\pi \gamma_{*} r_{b}}\right)^{4} \frac{\gamma_{*}}{r_{b}} \mathcal{F}\left(\xi_{n}\right)$,

where the function $\mathcal{F}(\xi)$ is defined as $\mathcal{F}(\xi)=$ $\mathcal{F}_{+}(\xi)+\mathcal{F}_{-}(\xi)$ with

$$
\begin{aligned}
\mathcal{F}_{ \pm}\left(\xi_{n}\right)=\int_{0}^{\infty} & {\left[\frac{1-\xi K_{1}(\xi)}{\xi}\right]^{2} } \\
& \times\left[\frac{\left.1-\left|\xi \pm \xi_{n}\right|\right) K_{1}\left(\left|\xi \pm \xi_{n}\right|\right)}{\left|\xi \pm \xi_{n}\right|}\right]^{2} d \xi .
\end{aligned}
$$

$\mathcal{F}(\xi)$ is about constant over the interval $[-\infty, 1]$ where $\mathcal{F}(\xi) \simeq 0.11$. We conclude

$$
\begin{aligned}
\left\langle\left|\tilde{\rho}_{n}(s)\right|^{2}\right\rangle & =\left[\mathcal{G}^{(1)}\left(k_{n}\right)\right]^{2} C^{2} \tilde{\rho}^{2}\left[1+\frac{2}{\pi n_{b}}\left(\frac{I L_{s}}{I_{A} r_{b} \gamma \gamma_{*}}\right)^{2}\right. \\
& \left.\times\left(R_{56} C k_{n}\right)^{2} \frac{\gamma_{*}}{r_{b}} \frac{\xi_{n}^{2} \mathcal{F}\left(\xi_{n}\right)}{\left[1-\xi_{n} K_{1}\left(\xi_{n}\right)\right]^{2}}\right], \quad \text { (19) }
\end{aligned}
$$

yielding the following second-order accurate gain function [see Eq. (9)]

$$
\begin{aligned}
\mathcal{G}\left(k_{n}\right) & =\mathcal{G}^{(1)}\left(k_{n}\right)\left[1+\frac{2}{\pi n_{b}}\left(\frac{I L_{s}}{I_{A} r_{b} \gamma \gamma_{*}}\right)^{2}\right. \\
& \left.\times\left(R_{56} C k_{n}\right)^{2} \frac{\gamma_{*}}{r_{b}} \frac{\xi_{n}^{2} \mathcal{F}\left(\xi_{n}\right)}{\left[1-\xi_{n} K_{1}\left(\xi_{n}\right)\right]^{2}}\right]^{1 / 2} .
\end{aligned}
$$

\section{Numerical Example}

The gain function for the microbunching instability including nonlinear effects can be calculated by numerical solution of the Vlasov equation governing the evolution of the beam density function in phase space. In a Vlasov solver the beam density in phase space is represented on a grid. Shot noise is modelled by adding a random perturbation to a smooth initial beam density with an amplitude chosen so as to reproduce the expected shot-noise fluctuations present in the actual electron beam [8]. The main goal in this section is to provide an independent check of the analytical calculation of Sec. 4 by comparison against the numerical solutions obtained with a Vlasov solver $[9,8,10]$. We consider two case studies.

In Case study A we use the simplified model of dynamics underlying the analytical calculation reported in the previous sections. Collective effects (i.e. longitudinal space charge) are active only in the
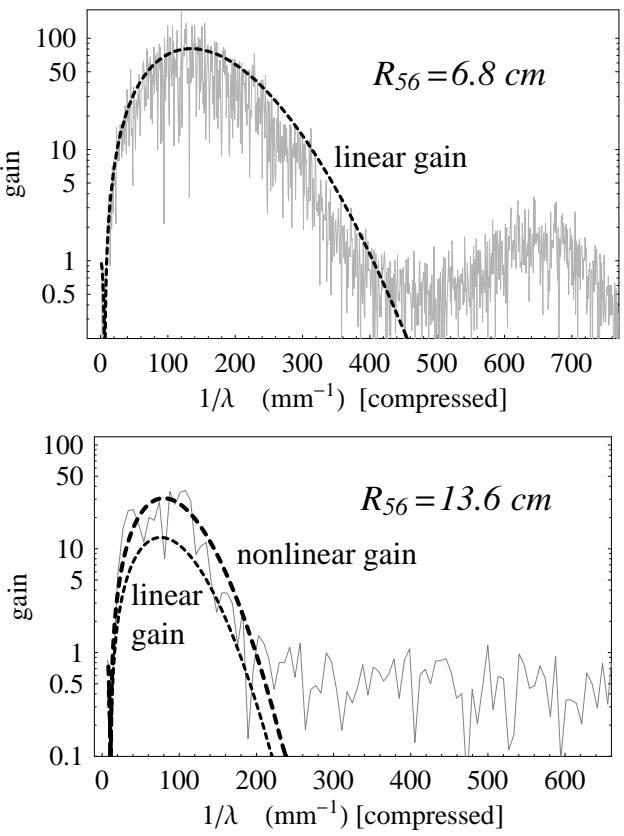

Fig. 1. Case study A (collective effects are active only in the straight section trailing the dispersive region of the lattice, as in the analytical model we have considered). The figure shows the microbunching gain function starting from shot noise as calculated by solving the Vlasov equation numerically (gray lines) and from theory (dashed lines). For an intermediate value of $R_{56}$ (top picture) the linear theory adequately reproduces the gain computed numerically. For larger $R_{56}$ (bottom picture) the second-order correct gain function gives a more accurate estimates than linear theory.

straight section preceding the dispersive region with the bunch compressor.

Case study B is for a more realistic setting where collective effects (including coherent synchrotron radiation, CSR) are active also in the dispersive region of the lattice.

In both cases the dispersive region spanning about $30 \mathrm{~m}$ length consists of two adjacent and identical 4dipole chicanes. The transfer matrix entry $R_{56}$ varies from zero to $6.8 \mathrm{~cm}$ by the end of the first chicane and to $13.6 \mathrm{~cm}$ by the end of the second. The beam energy chirp is tuned so as to result in compression factors $C=1.88$ and $C=16.2$ by the end of the first and the second chicane respectively. The initial peak current is $I=43 \mathrm{~A}$ corresponding to $N_{b}=$ $2.15 \times 10^{9}$ electrons over a length $L_{b}=2.4 \mathrm{~mm}$ or $n_{b}=9 \times 10^{11}$ particles $/ \mathrm{m}$ linear density.

The beam energy in the bunch compressor is $240 \mathrm{MeV}$. In Case study B the beam energy in the straight section varies from 41 to $240 \mathrm{MeV}$ over a distance of $L_{s}=48 \mathrm{~m}$. In Case study $\mathrm{A}$ the 

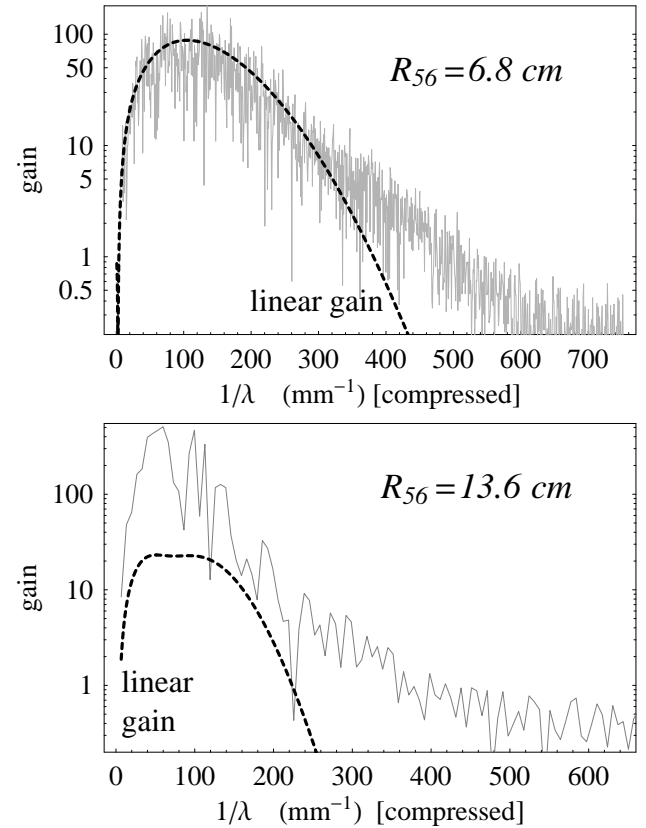

Fig. 2. Case study B (collective effects are active in both the straight section and the dispersive region of the lattice). At an intermediate $R_{56}$ (top picture) linear theory provides a good approximation for the gain as determined numerically (gray line) but for larger $R_{56}$ at the end of the second chicane (bottom picture) the actual gain is significantly larger.

beam energy is kept constant though the lattice at $240 \mathrm{MeV}$ with length of the straight section $L_{s}=83.6 \mathrm{~m}$ chosen so that the peak value of the linear gain through the first chicane (about 100) is comparable to that of Case study B. The initial energy spread in both cases is $5 \mathrm{keV}$ corresponding to $\sigma_{\delta}=2.1 \times 10^{-5}$ at $E=240 \mathrm{MeV}$.

The results of our calculation are shown in Fig. 1 and Fig. 2 for Case study A and B respectively. The gray curves represent the gain of the microbunching instability as determined numerically using the Vlasov solver. The gain is determined as $\left|\tilde{\rho}(s)_{n}\right| /(C \tilde{\rho})$, i.e. the ratio between the amplitude $\left|\tilde{\rho}(s)_{n}\right|$ of the Fourier mode of order $n$ calculated from the beam density at $s$ (for a single realization of the random perturbation) and the expected amplitude of the modes $\tilde{\rho}=\left\langle\left|\tilde{\rho}_{n}\left(s_{0}\right)\right|^{2}\right\rangle^{1 / 2}=\left(L_{b} \sqrt{N_{b}}\right)^{-1}$ at the start of the straight section $s_{0}$. As in Eq. (9) the factor $C$ normalizes the result to account for compression. The top an bottom pictures show the gain calculated when the beam exits the first and second chicane respectively.

The gain curves determined numerically by solving the Vlasov equation are contrasted to the gain curves from linear theory. In Fig. 1 we also make a comparison with the second-order accurate gain given by Eq. (20). Notice that the gain functions are plotted as functions of the inverse of the 'compressed' wavelength $\lambda^{-1}=C k / 2 \pi$, relative to the local density spectrum at $s$.

Linear theory appears to track quite well the gain calculated numerically through the first chicane while underestimating the gain at the exit of the second chicane. In Fig. 1 the second-order correct gain function is seen to give a better approximation, although arguably it could be slightly overestimating the gain, a possible indication that higher-order contributions may non be completely negligible.

In Case study B, (where account of collective effects is included in the dispersive region as well) not only the linear gain is larger (about a factor two in peak value) but the relative importance of the nonlinear corrections is also higher compared to Case study A. From Fig.2 we observe that the peak value of the gain calculated from the numerical solutions of the Vlasov equation is about an order of magnitude larger than the gain predicted by linear theory. Notice that in this picture we did not report the $2^{\text {nd }}$-order correction to the gain as the simplified model underlying the analytical formula does not properly describe the physical system considered for Case study B. The linear gain reported in Fig. 2 was calculated using the theory outlined in $[3,4,8]$ with inclusion of CSR in the bends.

\section{Conclusions}

Our main result is a demonstration that higher order contributions to the microbunching gain function beyond the linear approximation may be important when the noise present in the beam density at injection has a broad spectrum - as is the case for the beam density fluctuations stemming form shot noise.

A second-order correct theory for the gain function can be easily worked out for a simplified model of beam dynamics where energy modulations in the phase space of a relativistic beam are generated in nondispersive regions of the lattice. The analytical formula we have derived shows that the relative importance of the second order correction to the gain function scales quadratically with $R_{56}$ suggesting that decreasing $R_{56}$ could be a way to keep the nonlinear enhancement of the gain under control if this is found to be unacceptably large. 


\section{Acknowledgments}

Useful comments from and discussions with $\mathrm{Z}$. Huang, J. Qiang, and A. Zholents are gratefully acknowledged. Work supported by Department of Energy Contract No. DE-AC02-05CH11231.

\section{Appendix A. Statistics of Shot Noise}

Consider a subdivision of the beam length $L_{b}$ into $\mathcal{N}$ intervals of length $\Delta z=L_{b} / \mathcal{N}$. Denote with $N_{j}$ the population of electrons in the interval $z \in \Delta z[j-1, j]$. The occupation number $N_{j}$ is a random process obeying the Poisson statistics, which can be modelled as $N_{j}=\langle\Delta N\rangle+\langle\Delta N\rangle^{1 / 2} \eta_{j}$, where the average number of electrons $\langle\Delta N\rangle=\left\langle N_{j}\right\rangle$ in a interval $\Delta z$ is $\langle\Delta N\rangle=N_{b}\left(\Delta z / L_{b}\right)$ and $\eta_{j}$ is a univariate normal random process with vanishing average and variance equal to unity $\left\langle\eta_{j}\right\rangle=0$, $\left\langle\eta_{i} \eta_{j}\right\rangle=\delta_{i j}$. The last equation expresses the assumed lack of correlation in the fluctuations of the number of electrons populating different intervals. From $N_{b} \rho_{z}\left(z_{j} ; s_{0}\right) \Delta z=N_{j}$ it follows $\rho_{z}\left(z_{j} ; s_{0}\right)=$ $\rho_{0}\left[1+\eta_{j} /\langle\Delta N\rangle^{1 / 2}\right]$. For $j \neq j^{\prime}$ we have for the correlation $\left\langle\left[\rho_{z}\left(z_{j} ; s_{0}\right)-\rho_{0}\right]\left[\rho_{z}\left(z_{j^{\prime}} ; s_{0}\right)-\rho_{0}\right]\right\rangle=$ $\delta_{j j^{\prime}} \rho_{0}^{2} /\langle\Delta N\rangle=\left(N_{b} L_{b}\right)^{-1}\left(\delta_{j j^{\prime}} / \Delta z\right)$. In the limit $\Delta z \rightarrow 0$ the Kronecker is replaced by a Dirac delta $\left(\delta_{j j^{\prime}} / \Delta z\right) \rightarrow \delta\left(z-z^{\prime}\right)$.

\section{References}

[1] M. Borland, Y. Chae, P. Emma, J. Lewellen, V. Bharadwaj, W. Fawley, P. Krejcik, C. Limborg, S. Milton, H.-D. Nuhn, R. Soliday, and M. Woodley, Nucl. Instrum. Methods Phys. Res., Sect. A 483, 268 (2002).

[2] E.L. Saldin, E.A. Schneidmiller, and M.V. Yurkov, Nucl. Instrum. Methods Phys. Res., Sect. A 483, 516 (2002).

[3] S. Heifets, G. Stupakov, and S. Krinsky, Phys. Rev. ST Accel. Beams 5, 064401 (2002).

[4] Z. Huang and K.-J. Kim, Phys. Rev. ST Accel. Beams 5, 074401 (2002).

[5] E.L. Saldin, E.A. Schneidmiller, and M.V. Yurkov, Nucl. Instrum. Methods Phys. Res., Sect. A 528, 355 (2004)

[6] T. Shaftan and Z. Huang, Phys. Rev. ST Accel. Beams 7, 080702 (2004).

[7] Z. Huang, M. Borland, P. Emma, J. Wu, C. Limborg, G. Stupakov, and J. Welch, Phys. Rev. ST Accel. Beams 7, 074401 (2004)

[8] M. Venturini, Phys. Rev. ST Accel. Beams 10, 104401 (2007).

[9] M. Venturini, R. Warnock, and A. Zholents, Phys. Rev. ST Accel. Beams 10, 054403 (2007).

[10] M. Venturini and A. Zholents, Modeling Microbunching from Shot Noise Using Vlasov Solvers, to appear in NIM-A.
[11] J. Rosenzweig, C. Pellegrini, L. Serafini, C. Ternieden, and G. Travish, Nucl. Instrum. Methods Phys. Res., Sect. A 393, 376 (1997).

[12] M. Venturini, Phys. Rev. ST Accel. Beams 11, 034401 (2008)

\section{DISCLAIMER}

This document was prepared as an account of work sponsored by the United States Government. While this document is believed to contain correct information, neither the United States Government nor any agency thereof, nor The Regents of the University of California, nor any of their employees, makes any warranty, express or implied, or assumes any legal responsibility for the accuracy, completeness, or usefulness of any information, apparatus, product, or process disclosed, or represents that its use would not infringe privately owned rights. Reference herein to any specific commercial product, process, or service by its trade name, trademark, manufacturer, or otherwise, does not necessarily constitute or imply its endorsement, recommendation, or favoring by the United States Government or any agency thereof, or The Regents of the University of California. The views and opinions of authors expressed herein do not necessarily state or reflect those of the United States Government or any agency thereof , or The Regents of the University of California.

Ernest Orlando Lawrence Berkeley National Laboratory is an equal opportunity employer. 